\title{
Existence of Solutions for Generalized Vector Quasi-Equilibrium Problems by Scalarization Method with Applications
}

\author{
De-ning $Q \mathbf{u}^{1,2}$ and Cao-zong Cheng ${ }^{1}$ \\ ${ }^{1}$ College of Applied Science, Beijing University of Technology, Beijing 100124, China \\ ${ }^{2}$ College of Mathematics, Jilin Normal University, Siping, Jilin 136000, China \\ Correspondence should be addressed to De-ning Qu; qudening@126.com
}

Received 26 November 2012; Accepted 6 February 2013

Academic Editor: Ngai-Ching Wong

Copyright (c) 2013 D.-n. Qu and C.-z. Cheng. This is an open access article distributed under the Creative Commons Attribution License, which permits unrestricted use, distribution, and reproduction in any medium, provided the original work is properly cited.

The aim of this paper is to study generalized vector quasi-equilibrium problems (GVQEPs) by scalarization method in locally convex topological vector spaces. A general nonlinear scalarization function for set-valued mappings is introduced, its main properties are established, and some results on the existence of solutions of the GVQEPs are shown by utilizing the introduced scalarization function. Finally, a vector variational inclusion problem is discussed as an application of the results of GVQEPs.

\section{Introduction}

Recently, various vector equilibrium problems were investigated by adopting many different methods, such as the scalarization method (e.g., $[1,2])$, the recession method (e.g., [3]), and duality method (e.g., [4]).

The scalarization method is an important and efficacious tool of translating the vector problems into the scalar problems. In 1992, Chen [5] translated a vector variational inequality into a classical variational inequality by providing a kind of solution conceptions with variable domination structures. Gerth and Weidner [6] solved a vector optimization problem by introducing a scalarization function with a variable and Gong [1] dealt with vector equilibrium problems by using the scalarization function defined in [6]. By constructing new nonlinear scalarization functions with two variables, Chen and Yang [7] discussed a vector variational inequality and Chen et al. [2] investigated a generalized vector quasi-equilibrium problem (GVQEP), respectively. In addition, the authors in $[8,9]$ studied the systems of vector equilibrium problems by the scalarization method since the gap functions, indeed established by the nonlinear scalarization function defined in [2], were adopted.

In this paper, we will discuss the GVQEPs by utilizing scalarization method. The essential preliminaries are listed in Section 2. On the basis of the works in $[2,6,7]$, a general nonlinear scalarization function of a set-valued mapping is produced under a variable ordering structure and its main properties are discussed in Section 3. The results of properties for the general nonlinear scalarization function generalized the corresponding ones in [2]. In Section 4, some results on the existence of solutions of the GVQEPs are proved by employing the scalarization function introduced in Section 3. The GVQEPs are different from the one in [10] and include the one in [2] as a special case. It is worth mentioning that the existence results of solutions for the GVQEPs extend the corresponding one in [2]. Finally, a vector variational inclusion problem (VVIP) is given as an application of the GVQEPs in Section 5.

Suppose that $X$ and $Y$ are topological vector spaces (TVSs). The subset $D \subset Y$ is called a cone, if $\lambda x \in D$ for all $x \in D$ and $\lambda>0$. A cone $D \subset Y$ is said to be proper, if $D \neq Y$. $A$ is called $D$-closed [11] if $A+K$ is closed and $D$-bonded [11] if for each neighborhood $U$ of zero in $Y$, there exists $\lambda>0$ such that $A \subset U+D$. A set-valued mapping $G: X \rightarrow 2^{Y}$ is called strict, if $G(x) \neq \emptyset$ for any $x \in X$. Throughout this paper, $\mathbb{R}$ denotes by the set of the real numbers. Several notations are also listed as follows:

$$
G(A)=\bigcup_{u \in A} G(u),
$$




$$
\begin{gathered}
x-B=\{x-y: y \in B\}, \\
A-B=\{x-y: x \in A, y \in B\},
\end{gathered}
$$

where $A, B \subset X$ are nonempty, $x \in X$ and $G: X \rightarrow 2^{Y}$. Incidentally, every TVS is Hausdorff (see [12]).

\section{Preliminaries}

Let $X$ and $Y$ be topological spaces and $E \subset X$ a nonempty subset. A function $g: X \rightarrow \mathbb{R}$ is said to be upper semicontinuous (usc for brevity) on $X$, if $\{u \in X: g(u)<\lambda\}$ is open for each $\lambda \in \mathbb{R}$; to be lower semicontinuous (lsc for brevity), $\{u \in X: g(u)>\lambda\}$ is open for each $\lambda \in \mathbb{R}$. In addition, some known notions of continuity and closeness for a set-valued mapping are given (see [13]). A set-valued mapping $G: E \rightarrow 2^{Y}$ is said to be $u s c$ at $u_{0} \in E$, if for any neighborhood $N\left(G\left(u_{0}\right)\right)$ of $G\left(u_{0}\right)$, there exists a neighborhood $B\left(u_{0}\right)$ of $u_{0}$ such that $G(u) \subset N\left(G\left(u_{0}\right)\right)$ for all $u \in B\left(u_{0}\right)$; to be $l s c$ at $u_{0} \in E$, if for any $y_{0} \in G\left(u_{0}\right)$ and any neighborhood $N\left(y_{0}\right)$ of $y_{0}$, there exists a neighborhood $B\left(u_{0}\right)$ of $u_{0}$ such that $G(u) \cap N\left(y_{0}\right) \neq \emptyset$ for all $u \in B\left(u_{0}\right)$; to be $u s c$ (resp., lsc) on $E$, if $G$ is usc (resp., lsc) at each $u \in E$; to be continuous at $u_{0} \in E$ (resp., on $E$ ), if $G$ is usc and lsc at $u_{0}$ (resp., on $E$ ); to be closed, if its graph $\operatorname{Graph}(G)=\{(u, y) \in$ $E \times Y: y \in G(u)\}$ is closed in $E \times Y$.

Let $Y$ and $Z$ be real TVSs, $A \subset Y$ a nonempty subset, and $D$ a convex cone in $Y . \widehat{y} \in A$ is called, vector minimal point (resp., weakly vector minimal point) of $A$, if $y-\widehat{y} \notin-D \backslash\{0\}$ (resp., $y-\widehat{y} \notin-$ int $D$ ) for each $y \in A$. The set of vector minimal points (resp., weakly vector minimal points) of $A$ is denoted by $\operatorname{Min}_{D} A$ (resp., $w \operatorname{Min}_{D} A$ ).

Definition 1. Let $B \subset Z$ be nonempty convex subset. $G: B \rightarrow$ $2^{Y}$ is called generalized $D$-quasiconvex, if for any $y \in Y$, the set $\{u \in B: G(u) \subset y-D\}$ is convex (here, $\emptyset$ is regarded as a convex set).

The generalized $D$-quasiconvexity and the $D$-quasiconvexity introduced in [14] for the set-valued mappings are both generalizations of $D$-quasiconvexity for the single-valued mapping introduced in [11], but they are indeed distinct. See the following example.

Example 2. Let $Y=\mathbb{R}^{2}, Z=B=\mathbb{R}$, and $D=\mathbb{R}_{+}^{2}$ and define $G_{1}, G_{2}: B \rightarrow 2^{Y}$ as

$$
\begin{gathered}
G_{1}(u)=\left\{\left(x_{1}, x_{2}\right): x_{1}=u,-u^{2} \leq x_{2} \leq 0\right\}, \\
G_{2}(u)=\left\{\left(x_{1}, x_{2}\right): x_{1}=u, 0 \leq x_{2} \leq|\sin u|\right\} .
\end{gathered}
$$

Then $G_{1}$ is generalized $D$-quasiconvex but not $D$-quasiconvex, while $G_{2}$ is just the reverse. Indeed, for any fixed $y=$ $\left(x_{1}, x_{2}\right) \in Y$, both the sets

$$
\left\{u \in B: G_{1}(u) \subset y-D\right\}=\left\{\begin{array}{l}
\emptyset, \\
\text { if } x_{2}<0, x_{1} \in \mathbb{R}, \\
\left(-\infty, x_{1}\right] \\
\text { otherwise, }
\end{array}\right.
$$

$\left\{u \in B: G_{2}(u) \cap(y-D) \neq \emptyset\right\}=\left\{\begin{array}{l}\emptyset, \\ \text { if } x_{2}<0, x_{1} \in \mathbb{R}, \\ \left(-\infty, x_{1}\right], \\ \text { otherwise }\end{array}\right.$

are convex. But the set

$$
\left\{u \in \mathrm{B}: G_{1}(u) \cap((2,-1)-D) \neq \emptyset\right\}=(-\infty,-1] \cup[1,2]
$$

is not convex and neither is the set

$$
\left\{u \in B: G_{2}(u) \subset(0,0)-D\right\}=\{-n \pi: n=0,1,2, \ldots\} .
$$

Lemma 3 (see [15]). Let $X$ and $Y$ be topological spaces and $S: X \rightarrow 2^{Y}$ a set-valued mapping.

(1) If $S$ is usc with closed values, then $S$ is closed.

(2) If $X$ is compact and $S$ is usc with compact values, then $S(X)$ is compact.

Lemma 4 (see [13]). Let $X$ and $Y$ be Hausdorff topological spaces and $E \subset X$ a nonempty compact set and let $h: E \times Y \rightarrow$ $\mathbb{R}$ be a function and $G: E \rightarrow 2^{Y}$ a set-valued mapping. If $h$ is continuous on $E \times Y$ and $G$ is continuous with compact values, then the marginal function $V(u)=\sup _{y \in G(u)} h(u, y)$ is continuous and the marginal set-valued mapping $\delta(u)=\{y \in$ $G(u): V(u)=h(u, y)\}$ is usc.

Lemma 5 (Kakutani, see [13, Theorem 13 in Section 4 Chapter 6]). Let $E$ be a nonempty compact and convex subset of a locally convex TVS X. If $\Phi: E \rightarrow 2^{E}$ is usc and $\Phi(x)$ is a nonempty, convex, and closed subset for any $x \in E$, then there exists $\bar{x} \in E$ such that $\bar{x} \in \Phi(\bar{x})$.

\section{A General Nonlinear Scalarization Function}

From now on, unless otherwise specified, let $X, Y$, and $Z$ be real TVSs and $E \subset X$ and $F \subset Z$ nonempty subsets. Let $C$ : $E \rightarrow 2^{Y}$ be a set-valued mapping such that for any $x \in E$, $C(x)$ is a proper, closed, and convex cone with int $C(x) \neq \emptyset$.

In this section, suppose that $G: F \rightarrow 2^{Y}$ is a strict mapping with compact values and $e: E \rightarrow Y$ is a vector-valued mapping with $e(x) \in \operatorname{int} C(x)$, for all $x \in$ E. Obviously, $G(u)$ is $C(x)$-closed [11, Definition 3.1 and Proposition 3.3] for each $x \in E$ and $u \in F$. Then, in view of [16, Lemma 3.1], we can define a general nonlinear scalarization function of $\mathrm{G}$ as follows.

Definition 6. The general nonlinear scalarization function $\xi_{G}: E \times F \rightarrow \mathbb{R}$, of $G$ is defined as

$$
\begin{array}{r}
\xi_{G}(x, u)=\min \{\lambda \in \mathbb{R}: G(u) \subset \lambda e(x)-C(x)\}, \\
\forall(x, u) \in E \times F .
\end{array}
$$

Remark 7. If $X=Y=Z=E=F$ and $G(u)=\{u\}$, for all $u \in$ $F$, then the general nonlinear scalarization function $\xi_{G}$ of $G$ becomes the nonlinear scalarization function defined in [2]. 
Example 8. Let $X=Z=\mathbb{R}, Y=\mathbb{R}^{2}$, and $E=F=[0,+\infty) \subset$ $X$ and let $G, C: E \rightarrow 2^{Y}, e: E \rightarrow Y$ define as

$$
\begin{array}{cc}
G(u)=\{(U, V): U-u \leq V \leq u-U, 0 \leq U \leq u\}, & \forall u \in F, \\
C(x)=\left\{(U, V): 0 \leq V \leq\left(\frac{3}{2}+x\right) U, U \geq 0\right\}, & \forall x \in E, \\
e(x)=(1,1), \quad \forall x \in E, &
\end{array}
$$

respectively. We can see that $\xi_{G}(x, u)=(1+(2 /(1+2 x))) u$.

Definition 9. Let $D \subset Y$ be a cone, $H: F \rightarrow 2^{Y}$, and $\varsigma: F \rightarrow$ $\mathbb{R}$. $\varsigma$ is called monotone (resp., strictly monotone) with respect to ( $w r t$ for brevity) $H$, if for any $u_{1}, u_{2} \in F, H\left(u_{1}\right)-H\left(u_{2}\right) \subset$ int $D$ implies that $\varsigma\left(u_{2}\right) \leq \varsigma\left(u_{1}\right)$ (resp., $\varsigma\left(u_{2}\right)<\varsigma\left(u_{1}\right)$ ).

Obviously, the strict monotonicity wrt $H$ implies the monotonicity wrt $H$. Moreover, if $H(u)=\{u\}$, for all $u \in F$, then the (strict) monotonicity wrt $H$ of $\varsigma$ is equivalent to the (strict) monotonicity under the general order structures. The following examples illuminate the relationship between the monotonicity wrt $H$ and the monotonicity in the normal sense when $H$ is not the identity mapping.

Example 10. Let $Y=Z=\mathbb{R}, F=[0,10]$, and $D=\mathbb{R}_{+}$and let

$$
\begin{gathered}
\varsigma(u)= \begin{cases}u, & u \in[0,5], \\
0, & u \in(5,10],\end{cases} \\
H(u)= \begin{cases}{[u, u+1],} & u \in[0,5), \\
{[0,6],} & u=5, \\
{[0,10-u],} & u \in(5,10] .\end{cases}
\end{gathered}
$$

Then $H\left(u_{1}\right)-H\left(u_{2}\right) \subset$ int $D$ implies that $\varsigma\left(u_{1}\right)>\varsigma\left(u_{2}\right)$. Thus $\varsigma$ is strictly monotone wrt $H$, while $\varsigma$ is not monotone in the normal sense.

Example 11. Let $Y=Z=\mathbb{R}, F=[0,5]$, and $D=\mathbb{R}_{+}$and let $\varsigma(u)=u$, for all $u \in F$, and $H(u)=[-u-1,-u]$, for all $u \in F$. Then $\varsigma$ is strictly monotone in the normal sense, but $\varsigma$ is not monotone wrt $H$. As the case stands, $H\left(u_{1}\right)-H\left(u_{2}\right) \subset$ int $D$ is equivalent to $u_{1}, u_{2} \in F$ and $u_{2}-u_{1}>1$, which just results in $\varsigma\left(u_{1}\right)<\varsigma\left(u_{2}\right)$.

Now some main properties of the general nonlinear scalarization function are established. First, according to [16, Proposition 3.1], we have the following.

Theorem 12. For each $\lambda \in \mathbb{R}, x \in E$ and $u \in F$, the following assertions hold.

(1) $\xi_{G}(x, u)<\lambda \Leftrightarrow G(u) \subset \lambda e(x)-\operatorname{int} C(x)$.

(2) $\xi_{G}(x, u) \leq \lambda \Leftrightarrow G(u) \subset \lambda e(x)-C(x)$.

Theorem 13. $\xi_{G}$ is strictly monotone wrt $G$ in the second variable.
Proof. Letting $u_{1}, u_{2} \in F$ such that $G\left(u_{1}\right)-G\left(u_{2}\right) \subset \operatorname{int} C(x)$ and $\lambda=\xi_{G}\left(x, u_{1}\right)$, we have

$$
\begin{aligned}
G\left(u_{2}\right) & \subset G\left(u_{1}\right)-\operatorname{int} C(x) \\
& \subset \lambda e(x)-C(x)-\operatorname{int} C(x) \quad(\text { by Definition 6) } \\
& \subset \lambda e(x)-\operatorname{int} C(x)
\end{aligned}
$$

It is from this assertion that $\xi_{G}\left(x, u_{2}\right)<\lambda=\xi_{G}\left(x, u_{1}\right)$ by Theorem 12 (1).

Theorem 14. Suppose that e is continuous.

(1) If $W$ and $G$ are usc on $E$ and $F$, respectively, where

$$
W(x)=Y \backslash \operatorname{int} C(x), \quad \forall x \in E,
$$

then $\xi_{G}$ is usc on $E \times F$.

(2) If $C$ is usc on $E$ and $G$ is lsc on $F$, then $\xi_{G}$ is lsc on $E \times F$.

Proof. (1) It is sufficient to attest the fact that for each $\lambda \in \mathbb{R}$, the set

$$
A=\left\{(x, u) \in E \times F: \xi_{G}(x, u) \geq \lambda\right\}
$$

is closed. As a matter of fact, for any sequence $\left\{\left(x_{k}, u_{k}\right)\right\}$ in $A$ such that $\left(x_{k}, u_{k}\right) \rightarrow\left(x_{0}, u_{0}\right)$ as $k \rightarrow \infty, \xi_{G}\left(x_{k}, u_{k}\right) \geq$ $\lambda$, that is, $G\left(u_{k}\right) \not \subset \lambda e\left(x_{k}\right)-\operatorname{int} C\left(x_{k}\right)$, by Theorem $12(1)$. Obviously, $F_{0}=\left\{\bar{u}, u_{1}, u_{2}, \ldots\right\} \subset F$ is compact and so is $G\left(F_{0}\right)$ by Lemma $3(2)$. Then there exists $y_{k} \in G\left(u_{k}\right) \subset G\left(F_{0}\right)$ such that $y_{k} \notin \lambda e\left(x_{k}\right)-\operatorname{int} C\left(x_{k}\right)$ and a subsequence $\left\{y_{k_{i}}\right\}$ of $\left\{y_{k}\right\}$, such that $y_{k_{i}} \rightarrow y_{0} \in G\left(F_{0}\right)$ as $i \rightarrow \infty$ and

$$
\begin{gathered}
\lambda e\left(x_{k_{i}}\right)-y_{k_{i}} \notin \operatorname{int} C\left(x_{k_{i}}\right), \\
\text { that is, } \lambda e\left(x_{k_{i}}\right)-y_{k_{i}} \in W\left(x_{k_{i}}\right) .
\end{gathered}
$$

Obviously, $G$ is usc with closed values, which implies that $G$ is closed by Lemma $3(1)$. Hence, $y_{0} \in G\left(u_{0}\right)$. Similarly, $W$ is closed. Letting $i \rightarrow \infty$ in (12) and applying the continuity of $e$ and the closeness of $W$, we obtain that $\lambda e\left(x_{0}\right)-y_{0} \epsilon$ $W\left(x_{0}\right)$. Namely, $y_{0} \notin \lambda e\left(x_{0}\right)-\operatorname{int} C\left(x_{0}\right)$. Thus $G\left(u_{0}\right) \not \subset$ $\lambda e\left(x_{0}\right)-\operatorname{int} C\left(x_{0}\right)$, which is equivalent to $\xi_{G}\left(x_{0}, u_{0}\right) \geq \lambda$ by Theorem 12 (1). Therefore, $\lambda \in A$ and $A$ is closed.

(2) It's enough to argue that for each $\lambda \in \mathbb{R}$, the set

$$
B=\left\{(x, u) \in E \times F: \xi_{G}(x, u) \leq \lambda\right\}
$$

is closed. In fact, for any sequence $\left\{\left(x_{k}, u_{k}\right)\right\}$ in $B$ such that $\left(x_{k}, u_{k}\right) \rightarrow\left(x_{0}, u_{0}\right)$ as $k \rightarrow \infty$, by Theorem $12(2)$, $\xi_{G}\left(x_{k}, u_{k}\right) \leq \lambda$, equivalently, $G\left(u_{k}\right) \subset \lambda e\left(x_{k}\right)-C\left(x_{k}\right)$. For any $y_{0} \in G\left(u_{0}\right)$, there exists $y_{k} \in G\left(u_{k}\right)$ such that $y_{k} \rightarrow y_{0}$ as $k \rightarrow \infty$ according to the equivalent definition of lower semicontinuity of $G$ (see [13], page 108). Also,

$$
\lambda e\left(x_{k}\right)-y_{k} \in C\left(x_{k}\right) \text {. }
$$

Linking the continuity of $e$ with the closeness of $C$ inferred from Lemma 3 (1) and letting $k \rightarrow \infty$ in (14), we get $\lambda e\left(x_{0}\right)-$ $y_{0} \in C\left(x_{0}\right)$ and $G\left(u_{0}\right) \subset \lambda e\left(x_{0}\right)-C\left(x_{0}\right)$. Consequently, 
Theorem 12 (2) leads to $\xi_{G}\left(x_{0}, u_{0}\right) \leq \lambda$ which amounts to $\left(x_{0}, u_{0}\right) \in B . B$ is closed.

If $X=Y=Z=E=F$ ( $F$ is not required to be compact) and $G(u)=\{u\}$, for all $u \in F$, then Theorem 14 becomes [2, Theorem 2.1]. Actually, [2, Theorem 2.1] can be regarded as the case where $G$ is continuous in Theorem 14 . In addition, Example 2.1 (resp., Example 2.2) in [2] shows that if $C$ (resp., $W$ ) is not usc, maybe the general nonlinear scalarization function fails to be lsc (resp., usc) under all the other assumptions. Now the following example demonstrates that the assumption of the upper semicontinuity (resp., lower semicontinuity) of $G$ is necessary in Theorem 14 (1) (resp., (2)) even if $C$ is continuous.

Example 15. Let $X=Z=\mathbb{R}, Y=\mathbb{R}^{2}, E=\mathbb{R}_{+}$, and $F=$ $[-10,10] \subset Z$, and let

$$
\begin{gathered}
e(x)=(1,1), \quad \forall x \in E \\
C(x)=\left\{(U, V): 0 \leq V \leq\left(\frac{3}{2}+x\right) U, U \geq 0\right\}, \quad \forall x \in E .
\end{gathered}
$$

(1) Define

$$
G(u)= \begin{cases}{[u, u+1],} & u \in(0,10], \\ 0, & u \in[-10,0] .\end{cases}
$$

Evidently, $G$ is not usc on $F$. After simply calculating,

$$
\xi_{G}(x, u)= \begin{cases}u+1, & x \in E, 0<u \leq 10, \\ 0, & x \in E,-10 \leq u \leq 0 .\end{cases}
$$

$\xi_{G}$ is not usc on $E \times F$ due to the fact that $\{(x, u) \in E \times F$ : $\xi(x, u) \geq 1\}=\mathbb{R}_{+} \times(0,10]$ is not closed.

(2) Consider the following mapping:

$$
G(u)= \begin{cases}{[u, u+1],} & u \in[0,10], \\ 0, & u \in[-10,0) .\end{cases}
$$

Obviously, $G$ is not lsc on $F$. Also, $\xi_{G}$ fails to be lsc on $E \times F$, where

$$
\xi_{G}(x, u)= \begin{cases}u+1, & x \in E, 0 \leq u \leq 10 \\ 0, & x \in E,-10 \leq u<0\end{cases}
$$

\section{Existence Results on Solutions of the GVQEPs}

In this section, further suppose that $X, Y$, and $Z$ are locally convex. Let $g: F \rightarrow E$ be a vector-valued mapping. Now two GVQEPs are characterized as follows:

GVQEP1: Seek $\bar{x} \in P(\bar{x})$ and $\bar{z} \in Q(\bar{x})$ such that

$$
\begin{aligned}
& f(v, \bar{z})-f(\bar{x}, \bar{z}) \\
& \quad \not \subset-\operatorname{int} C(g(\bar{z})), \quad \forall v \in P(\bar{x}),
\end{aligned}
$$

where $P: E \rightarrow 2^{E}, Q: E \rightarrow 2^{F}$, and $f: E \times F \rightarrow$ $2^{Y}$ are set-valued mappings.
GVQEP2: Find $\bar{x} \in P(\bar{x})$ and $\bar{z} \in Q(\bar{x})$ such that

$$
\begin{aligned}
& f(v, \bar{x}, \bar{z})-f(\bar{x}, \bar{x}, \bar{z}) \\
& \not \subset-\operatorname{int} C(g(\bar{z})), \quad \forall v \in P(\bar{x}),
\end{aligned}
$$

where $P: E \rightarrow 2^{E}, Q: E \rightarrow 2^{F}$, and $f: E \times E \times F \rightarrow$ $2^{Y}$ are set-valued mappings.

It's worth noting that the GVQEP considered in [2] is just the special case of the GVQEP2 (when $f$ is single valued).

Lemma 16. Let $e: E \rightarrow Y$ be a vector-valued mapping such that $e(x) \in \operatorname{int} C(x)$ for all $x \in E$ and $f: E \times F \rightarrow 2^{Y}$ a set-valued mapping and define $f_{z}(x)=f(x, z)$ for each $z \in F$. Iffor each $z \in F, f_{z}$ is generalized $C(g(z))$-quasiconvex with compact values, then $x \mapsto h(x, z)$ is $\mathbb{R}_{+}$-quasiconvex, where $h(x, z)=\xi_{f_{z}}(g(z), x)$ and $\xi_{f_{z}}$ is the general nonlinear scalarization function of $f_{z}$.

Proof. It's sufficient to testify that $L_{z}(\lambda) \subset X$ is convex, where

$$
L_{z}(\lambda)=\left\{x \in E: \xi_{f_{z}}(g(z), x) \leq \lambda\right\}
$$

for each $z \in F$ and $\lambda \in \mathbb{R}$. Indeed, for any $x_{1}, x_{2} \in L_{z}(\lambda)$,

$$
\xi_{f_{z}}\left(g(z), x_{i}\right) \leq \lambda, \quad i=1,2,
$$

that is, $f_{z}\left(x_{i}\right) \subset \lambda e(g(z))-C(g(z)), \quad i=1,2$.

Clearly, for each $z \in E, x_{1}, x_{2} \in M$, where

$$
M=\left\{x \in E: f_{z}(x) \subset \lambda e(g(z))-C(g(z))\right\} .
$$

$M$ is convex by reason of the generalized $C(g(z))$ quasiconvexity of $f_{z}$ and so $\tilde{x}=t x_{1}+(1-t) x_{2} \in M$ for all $0 \leq t \leq 1$. Hence,

$$
f_{z}(\tilde{x}) \subset \lambda e(g(z))-C(g(z))
$$

which is equal to $\xi_{f_{z}}(g(z), \tilde{x}) \leq \lambda$ by Theorem 12 (2). Thus, $\tilde{x} \in L_{z}(\lambda)$ and $L_{z}(\lambda)$ is convex.

Now a result on existence of solutions of the GVQEP1 is verified by making use of the general nonlinear scalarization function defined in Section 3.

Theorem 17. Let $E$ and $F$ be compact and convex subsets and $P: E \rightarrow 2^{E}, Q: E \rightarrow 2^{F}$, and $f: E \times F \rightarrow 2^{Y}$ set-valued mappings. For each $z \in F$, define $f_{z}(x)=f(x, z)$. Suppose that the following conditions are fulfilled:

(a) $x \mapsto \operatorname{int} C(x)$ has a continuous select $e: E \rightarrow Y$;

(b) both $C$ and $W$ are usc on $E$, where

$$
W(x)=Y \backslash \operatorname{int} C(x), \quad \forall x \in E ;
$$

(c) $g$ is continuous, $f$ and $P$ are strict and continuous, and $Q$ is strict and usc;

(d) for each $z \in F, f_{z}$ is generalized $C(g(z))$-quasiconvex; 
(e) for each $(x, z) \in E \times F, f(x, z)$ is compact and for each $x \in E$, both $P(x)$ and $Q(x)$ are closed and convex.

Then the GVQEP1 has a solution $(\bar{x}, \bar{z}) \in E \times F$; that is, there exist $\bar{x} \in P(\bar{x})$ and $\bar{z} \in Q(\bar{x})$ such that

$$
f(v, \bar{z})-f(\bar{x}, \bar{z}) \not \subset-\operatorname{int} C(g(\bar{z})), \quad \forall v \in P(\bar{x}) .
$$

Further assume that $f(x, z) \subset C(g(z))$ for each $x \in E$ and $z \in Q(x)$. Then

$$
f(v, \bar{z}) \not \subset-\operatorname{int} C(g(\bar{z})), \quad \forall v \in P(\bar{x}) .
$$

Proof. Denote $K=E \times F$ and $w=(x, z)$ where $x \in E$ and $z \in F$, and define $\widetilde{g}: K \rightarrow E$ as

$$
\widetilde{g}(w)=\tilde{g}(x, z)=g(z) .
$$

Obviously, $\tilde{g}$ is continuous on $K$. The general nonlinear scalarization function of $f$

$$
\xi_{f}(u, w)=\min \{\lambda \in \mathbb{R}: f(w) \subset \lambda e(u)-C(u)\}
$$

is continuous on $E \times K$ by Theorem 14. Set $h(x, z)=$ $\xi_{f_{z}}(g(z), x)$, where $\xi_{f_{z}}$ is the general nonlinear scalarization function of $f_{z}$. Since

$$
\begin{aligned}
h(w) & =h(x, z) \\
& =\min \left\{\lambda \in \mathbb{R}: f_{z}(x) \subset \lambda e(g(z))-C(g(z))\right\} \\
& =\min \{\lambda \in \mathbb{R}: f(x, z) \subset \lambda e(\widetilde{g}(x, z))-C(\widetilde{g}(x, z))\} \\
& =\min \{\lambda \in \mathbb{R}: f(w) \subset \lambda e(\widetilde{g}(w))-C(\widetilde{g}(w))\} \\
& =\xi_{f}(\widetilde{g}(w), w),
\end{aligned}
$$

$h$ is continuous on $K$ by virtue of the continuity of $\xi_{f}$ and $\widetilde{g}$.

Define $\widetilde{P}: E \times F \rightarrow 2^{E \times F}$ as

$$
\widetilde{P}(x, z)=P(x) \times\{z\}, \quad \forall(x, z) \in E \times F .
$$

Obviously, $\widetilde{P}$ has compact values and is continuous. Define a set-valued mapping $\delta: E \times F \rightarrow 2^{E \times F}$ as

$$
\begin{aligned}
\delta(x, z) & =\left\{(u, z) \in P(x) \times\{z\}: h(u, z)=\min _{v \in P(x)} h(v, z)\right\} \\
& =\left\{(u, z) \in P(x) \times\{z\}:-h(u, z)=\max _{v \in P(x)}-h(v, z)\right\} \\
& =\left\{(u, a) \in \widetilde{P}(x, z):-h(u, a)=\max _{(v, b) \in \widetilde{P}(x, z)}-h(v, b)\right\} .
\end{aligned}
$$

By Lemma $4, \delta(x, z)$ is usc. Let

$$
\eta(x, z)=\left\{u \in P(x): h(u, z)=\min _{v \in P(x)} h(v, z)\right\} .
$$

Then $\delta(x, z)=\eta(x, z) \times\{z\}$. The upper semicontinuity of $\eta$ is obvious. Now we show that for each $(x, z) \in E \times F, \eta(x, z)$ is convex and closed. Detailedly, (i) Define

$$
\lambda=\min _{v \in P(x)} h(v, z)
$$

Then for any $u_{1}, u_{2} \in \eta(x, z), h\left(u_{i}, z\right)=\lambda, i=1,2$. Clearly, $\tilde{u}=t u_{1}+(1-t) u_{2} \in P(x)$ for all $t \in[0,1]$ since $P(x)$ is convex. In view of condition (d) and Lemma 16, $x \mapsto h(x, z)$ is $\mathbb{R}_{+}$-quasiconvex, which deduces that for each $z \in F$, the set

$$
L_{z}(\lambda)=\{x \in E: h(x, z) \leq \lambda\}
$$

is convex. Thus, $h(\widetilde{u}, z) \leq \lambda$. It follows from the definition of $\lambda$ that $h(\widetilde{u}, z)=\lambda$, which results in $\widetilde{u} \in \eta(x, z)$ and the convexity of $\eta(x, z)$.

(ii) For each $(x, z) \in E \times F$ and for any sequence $\left\{u_{k}\right\} \subset$ $\eta(x, z)$ such that $u_{k} \rightarrow u_{0}$ as $k \rightarrow \infty$,

$$
h\left(u_{k}, z\right)=\min _{v \in P(x)} h(v, z), \quad \forall k .
$$

Since $h$ is continuous,

$$
h\left(u_{0}, z\right)=\lim _{k \rightarrow \infty} h\left(u_{k}, z\right)=\min _{v \in P(x)} h(v, z) .
$$

Thus $u_{0} \in \eta(x, z)$ and $\eta(x, z)$ is closed.

Now consider a set-valued mapping $\Phi: E \times F \rightarrow 2^{E \times F}$ prescribed as

$$
\Phi(x, z)=\eta(x, z) \times Q(x), \quad \forall(x, z) \in E \times F .
$$

It's easy to check that $\Phi$ is usc on $E \times F$ and for each $(x, z) \epsilon$ $E \times F, \Phi(x, z)$ is convex and closed. By Lemma 5, $\Phi$ exists a fixed point $(\bar{x}, \bar{z})$, that is to say,

$$
\bar{x} \in P(\bar{x}), \quad \bar{z} \in Q(\bar{x}), \quad h(\bar{x}, \bar{z})=\min _{v \in P(\bar{x})} h(v, \bar{z}) .
$$

Hence, for each $v \in P(\bar{x}), h(v, \bar{z}) \geq h(\bar{x}, \bar{z})$. In other words,

$$
\xi_{f_{\bar{z}}}(g(\bar{z}), v) \geq \xi_{f_{\bar{z}}}(g(\bar{z}), \bar{x}),
$$

which deduces that $\bar{x} \in P(\bar{x}), \bar{z} \in Q(\bar{x})$, and

$$
\begin{aligned}
f(v, \bar{z})-f(\bar{x}, \bar{z}) & =f_{\bar{z}}(v)-f_{\bar{z}}(\bar{x}) \\
& \not \subset-\operatorname{int} C(g(\bar{z})), \quad \forall v \in P(\bar{x}),
\end{aligned}
$$

by Theorem 13. The first conclusion is proved. Further assume that $f(x, z) \subset C(g(z))$ for each $x \in E$ and $z \in Q(x)$. If the second conclusion is not true; namely, there exists $v \in P(\bar{x})$ such that $f(v, \bar{z}) \subset-\operatorname{int} C(g(\bar{z}))$, then

$$
\begin{aligned}
f(v, \bar{z})-f(\bar{x}, \bar{z}) & \subset-\operatorname{int} C(g(\bar{z}))-C(g(\bar{z})) \\
& =-\operatorname{int} C(g(\bar{z})) .
\end{aligned}
$$

This is absurd.

If mapping $C$ in Theorem 17 satisfies that for each $y \in Y$, $(\operatorname{int} C)^{-1}(y)=\{x \in E: y \in \operatorname{int} C(x)\}$ is open, then $x \mapsto \operatorname{int} C(x)$ must exist a continuous selection by Browder Selection Theorem [17]. Especially, when $f$ is single valued in Theorem 17, a result is stated as follows. 
Corollary 18. Let $E$ and $F$ be compact and convex subsets, $P$ : $E \rightarrow 2^{E}$ and $Q: E \rightarrow 2^{F}$ set-valued mappings and $f:$ $E \times F \rightarrow Y$ a vector-valued mapping. For each $z \in F$, define $f_{z}(x)=f(x, z)$. Suppose that the following conditions are in force:

(a) $x \mapsto$ int $C(x)$ has a continuous select $e: E \rightarrow Y$;

(b) both $C$ and $W$ are usc on $E$, where

$$
W(x)=Y \backslash \operatorname{int} C(x), \quad \forall x \in E ;
$$

(c) $f$ and $g$ are continuous, $P$ is strict and continuous, and $Q$ is strict and usc;

(d) for each $z \in F, f_{z}$ is $C(g(z))$-quasiconvex;

(e) for each $x \in E$, both $P(x)$ and $Q(x)$ are closed and convex.

Then there exist $\bar{x} \in P(\bar{x})$ and $\bar{z} \in Q(\bar{x})$ such that

$$
f(\bar{x}, \bar{z}) \in w \operatorname{Min}_{C(g(\bar{z}))} f(P(\bar{x}), \bar{z}) .
$$

Further suppose that $f(x, z) \in C(g(z))$ for each $x \in E$ and $z \in Q(x)$. Then

$$
f(P(\bar{x}), \bar{z}) \cap(-\operatorname{int} C(g(\bar{z})))=\emptyset .
$$

Theorem 19. Let $E$ and $F$ be compact and convex subsets and $P: E \rightarrow 2^{E}, Q: E \rightarrow 2^{F}$, and $f: E \times E \times F \rightarrow 2^{Y}$ set-valued mappings. For each $(x, z) \in E \times F$, define $f_{x z}(u)=f(u, x, z)$. Suppose that the following conditions hold:

(a) $x \mapsto \operatorname{int} C(x)$ has a continuous select $e: E \rightarrow Y$;

(b) both $C$ and $W$ are usc on $E$, where

$$
W(x)=Y \backslash \operatorname{int} C(x), \quad \forall x \in E ;
$$

(c) $g$ is continuous, $f$ and $P$ are strict and continuous, and $Q$ is strict and usc;

(d) for each $(x, z) \in E \times F, f_{x z}$ is generalized $C(g(z))$ quasiconvex;

(e) for each $(u, x, z) \in E \times E \times F, f(u, x, z)$ is compact and for each $x \in E$, both $P(x)$ and $Q(x)$ are closed and convex.

Then the GVQEP2 exists a solution $(\bar{x}, \bar{z}) \in E \times F$; namely, there exist $\bar{x} \in P(\bar{x})$ and $\bar{z} \in Q(\bar{x})$ such that

$$
f(v, \bar{x}, \bar{z})-f(\bar{x}, \bar{x}, \bar{z}) \not \subset-\operatorname{int} C(g(\bar{z})), \quad \forall v \in P(\bar{x}) .
$$

Furthermore, if $f(x, x, z) \subset C(g(z))$ for each $x \in E$ and $z \in$ $Q(x)$, then

$$
f(v, \bar{x}, \bar{z}) \not \subset-\operatorname{int} C(g(\bar{z})), \quad \forall v \in P(\bar{x}) .
$$

Proof. Denoting $K=E \times F$, we see that $K$ is compact. Define $\widetilde{g}: K \rightarrow E$ and $S: E \rightarrow 2^{K}$ as $\widetilde{g}(w)=$ $\tilde{g}(x, z)=g(z)$, for all $w=(x, z) \in K$ and $S(x)=\{x\} \times$ $\mathrm{Q}(x)$, for all $x \in E$, respectively. Then $\tilde{g}$ is continuous on $K$ and for each $w \in K, f_{w}$ is generalized $C(\tilde{g}(w))$-quasiconvex according to condition (d). Since $Q$ is strict and usc with compact values, so is $S$. Replacing $F, g$, and $Q$ in Theorem 17 by $K, \widetilde{g}$, and $S$, respectively, we see that these conclusions are true.
The result below follows from Theorem 19 immediately by further assuming that $f$ is single-valued.

Corollary 20 (see [2]). Let $E$ and $F$ be compact and convex subsets, $P: E \rightarrow 2^{E}$ and $Q: E \rightarrow 2^{F}$ set-valued mappings, and $f: E \times E \times F \rightarrow Y$ a vector-valued mapping. For each $(x, z) \in E \times F$, define $f_{x z}(u)=f(u, x, z)$. The following assumptions are in operation:

(a) $x \mapsto \operatorname{int} C(x)$ has a continuous select $e: E \rightarrow Y$;

(b) both $C$ and $W$ are usc on $E$, where

$$
W(x)=Y \backslash \operatorname{int} C(x), \quad \forall x \in E ;
$$

(c) $f$ and $g$ are continuous, $P$ is strict and continuous, and $Q$ is strict and usc;

(d) for each $(x, z) \in E \times F, f_{x z}$ is $C(g(z))$-quasiconvex;

(e) for each $x \in E$, both $P(x)$ and $Q(x)$ are closed and convex.

Then there exist $\bar{x} \in P(\bar{x})$ and $\bar{z} \in Q(\bar{x})$ such that

$$
f(\bar{x}, \bar{x}, \bar{z}) \in w \operatorname{Min}_{C(g(\bar{z}))} f(P(\bar{x}), \bar{x}, \bar{z}) .
$$

Additionally, on the assumption that $f(x, x, z) \in C(g(z))$ for each $x \in E$ and $z \in Q(x)$,

$$
f(P(\bar{x}), \bar{x}, \bar{z}) \cap(-\operatorname{int} C(g(\bar{z})))=\emptyset
$$

\section{An Application of GVQEP1: A VVIP}

Let $X$ be a real $F$-space ( $X$ is called an $F$-space [14], if it is a TVS such that its topology is induced by a complete invariant metric), $Y$ a real locally convex TVS, $E \subset X$ a nonempty subset and $D \subset Y$ a proper, closed, and convex cone with int $D \neq \emptyset$ and let $l: E \rightarrow 2^{L(X, Y)}$ be strict and continuous with compact values. A VVIP is described as follows: to find $\bar{x} \in E$ such that

$$
\langle l(\bar{x}), x-\bar{x}\rangle \not \subset-\operatorname{int} D, \quad \forall x \in E,
$$

where

$$
\langle l(z), x\rangle=\bigcup\{\langle p, x\rangle: p \in l(z)\}
$$

The VVIP was investigated in $[18,19]$. If $l$ is single valued, then the VVIP becomes a vector variational inequality problem, which was discussed in [20-22]. Note that $X$ is indeed locally convex.

Assume that $E$ is compact and convex and $\langle l(x), x\rangle$ is a singleton for each $x \in E$. 
Let

$$
\begin{gathered}
X=Z, \\
E=F, \\
C(x)=D, \quad \forall x \in E, \\
g: E \longrightarrow Y \text { be any continuous mapping, } \\
f(x, z)=\langle l(z), x\rangle, \quad \forall(x, z) \in E \times E, \\
P(x)=E, \quad \forall x \in E, \\
Q(x)=\{x\}, \quad \forall x \in E
\end{gathered}
$$

in Theorem 17. Then the GVQEP1 reduces to the VVIP. All the assumptions of Theorem 17 are verified as follows.

(a) and (b) Since $D$ is a constant cone, $x \mapsto$ int $D$ has a continuous selection and both $C$ and $W$ are usc.

(c) Obviously, $f$ is strict, $g$ is continuous, $P$ is strict and continuous, and $Q$ is strict and usc. In addition, $f$ is continuous with compact values.

In fact, for each fixed $\left(x_{0}, z_{0}\right) \in E \times E$, letting $m(p)=$ $\left\langle p, x_{0}\right\rangle$, we see that $m$ is continuous with compact values. So $f\left(x_{0}, z_{0}\right)=m\left(l\left(z_{0}\right)\right)$ is compact by the compactness of $l\left(z_{0}\right)$ and Lemma $3(2)$. Moreover, take $L(p, x, z)=\langle p, x\rangle$ and $H(x, z)=l(z)$. Obviously, $H$ is continuous with compact values. $L$ is continuous in view of the bilinearity of itself and [11, Theorem 2.17]. Thus

$$
f(x, z)=\bigcup_{p \in H(x, z)} L(p, x, z)
$$

is continuous by [13, Theorem 1$]$.

(d) For each $z \in E, x \mapsto f(x, z)$ is generalized $D$ quasiconvex by its linearity.

(e) The assertion that $f$ has compact values was verified in (c). Clearly, for each $x \in E$, both $P(x)$ and $Q(x)$ are closed and convex.

Thus there exist $(\bar{x}, \bar{z}) \in E \times E, \bar{x} \in P(\bar{x})=E$, and $\bar{z} \in$ $Q(\bar{x})=\{\bar{x}\}$ such that

$$
f(x, \bar{x})-f(\bar{x}, \bar{x}) \not \subset-\operatorname{int} D, \quad \forall x \in E,
$$

that is, $\langle l(\bar{x}), x\rangle-\langle l(\bar{x}), \bar{x}\rangle \not \subset-$ int $D, \quad \forall x \in E$.

Since $\langle l(x), x\rangle$ is a singleton for each $x \in E$,

$$
\langle l(\bar{x}), x-\bar{x}\rangle \not \subset-\operatorname{int} D, \quad \forall x \in E,
$$

which implies that $\bar{x}$ is a solution of VVIP.

Incidentally, the set-valued mapping $l$ satisfying the conditions above exists. For instance, let $X=\mathbb{R}^{2}, Y=\mathbb{R}$, and

$$
E=\left\{x=\left(x_{1}, x_{2}\right): x_{1}^{2}+x_{2}^{2} \leq 1\right\} \subset X .
$$

Define $l$ by

$$
l(x)=\left\{\left(\lambda x_{2},-\lambda x_{1}\right):|\lambda| \leq 1\right\}, \quad \forall x=\left(x_{1}, x_{2}\right) \in E .
$$

Clearly, $l$ is strict and continuous with compact values. Moreover, $\langle l(x), x\rangle=\{0\}$ for each $x \in E$.

\section{Acknowledgment}

The work was supported by both the Doctoral Fund of innovation of Beijing University of Technology (2012) and the 11th graduate students Technology Fund of Beijing University of Technology (No. ykj-2012-8236).

\section{References}

[1] X. H. Gong, "Scalarization and optimality conditions for vector equilibrium problems," Nonlinear Analysis: Theory, Methods \& Applications A, vol. 73, no. 11, pp. 3598-3612, 2010.

[2] G. Y. Chen, X. Q. Yang, and H. Yu, "A nonlinear scalarization function and generalized quasi-vector equilibrium problems," Journal of Global Optimization, vol. 32, no. 4, pp. 451-466, 2005.

[3] Q. H. Ansari and F. Flores-Bazán, "Recession methods for generalized vector equilibrium problems," Journal of Mathematical Analysis and Applications, vol. 321, no. 1, pp. 132-146, 2006.

[4] S. J. Li and P. Zhao, "A method of duality for a mixed vector equilibrium problem," Optimization Letters, vol. 4, no. 1, pp. 8596, 2010.

[5] G. Y. Chen, "Existence of solutions for a vector variational inequality: an extension of the Hartmann-Stampacchia theorem," Journal of Optimization Theory and Applications, vol. 74, no. 3, pp. 445-456, 1992.

[6] C. Gerth and P. Weidner, "Nonconvex separation theorems and some applications in vector optimization," Journal of Optimization Theory and Applications, vol. 67, no. 2, pp. 297320, 1990.

[7] G. Y. Chen and X. Q. Yang, "Characterizations of variable domination structures via nonlinear scalarization," Journal of Optimization Theory and Applications, vol. 112, no. 1, pp. 97-110, 2002.

[8] N. J. Huang, J. Li, and J. C. Yao, "Gap functions and existence of solutions for a system of vector equilibrium problems," Journal of Optimization Theory and Applications, vol. 133, no. 2, pp. 201212, 2007.

[9] J. Li and N. J. Huang, "An extension of gap functions for a system of vector equilibrium problems with applications to optimization problems," Journal of Global Optimization, vol. 39, no. 2, pp. 247-260, 2007.

[10] D. N. Qu and X. P. Ding, "Some quasi-equilibrium problems for multimaps," Journal of Sichuan Normal University. Natural Science Edition, vol. 30, no. 2, pp. 173-177, 2007.

[11] D. T. Luc, Theory of Vector Optimization, vol. 319 of Lecture Notes in Economics and Mathematical Systems, Springer, Berlin, Germany, 1989.

[12] W. Rudin, Functional Analysis, International Series in Pure and Applied Mathematics, McGraw-Hill, New York, NY, USA, 2nd edition, 1991.

[13] J. P. Aubin and I. Ekeland, Applied Nonlinear Analysis, John Wiley \& Sons, New York, NY, USA, 1984.

[14] L. J. Lin and Z. T. Yu, "On some equilibrium problems for multimaps," Journal of Computational and Applied Mathematics, vol. 129, no. 1-2, pp. 171-183, 2001.

[15] J. P. Aubin and A. Cellina, Differential Inclusion, Springer, Berlin, Germany, 1994.

[16] P. H. Sach and L. A. Tuan, "New scalarizing approach to the stability analysis in parametric generalized Ky Fan inequality problems," Journal of Optimization Theory and Applications, 2012. 
[17] F. E. Browder, "The fixed point theory of multi-valued mappings in topological vector spaces," Mathematische Annalen, vol. 177, pp. 283-301, 1968.

[18] G. M. Lee, D. S. Kim, B. S. Lee, and S. J. Cho, "Generalized vector variational inequality and fuzzy extension," Applied Mathematics Letters, vol. 6, no. 6, pp. 47-51, 1993.

[19] S. Park, B. S. Lee, and G. M. Lee, "A general vector-valued variational inequality and its fuzzy extension," International Journal of Mathematics and Mathematical Sciences, vol. 21, no. 4, pp. 637-642, 1998.

[20] F. Giannessi, "Theorems of alternative, quadratic programs and complementarity problems," in Variational Inequality Complementary Problems, R. W. Cottle, F. Giannessi, and J. L. Lions, Eds., pp. 151-186, John Wiley \& Sons, New York, NY, USA, 1980.

[21] X. Q. Yang, "Vector variational inequality and its duality," Nonlinear Analysis: Theory, Methods \& Applications A, vol. 21, no. 11, pp. 869-877, 1993.

[22] X. Q. Yang and C. J. Goh, "On vector variational inequalities: application to vector equilibria," Journal of Optimization Theory and Applications, vol. 95, no. 2, pp. 431-443, 1997. 


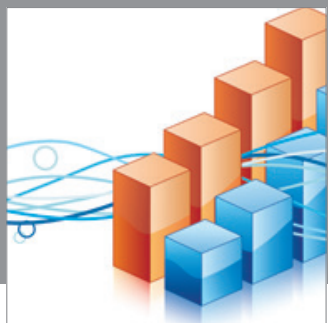

Advances in

Operations Research

mansans

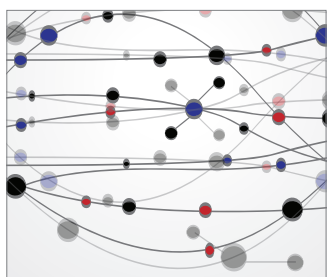

The Scientific World Journal
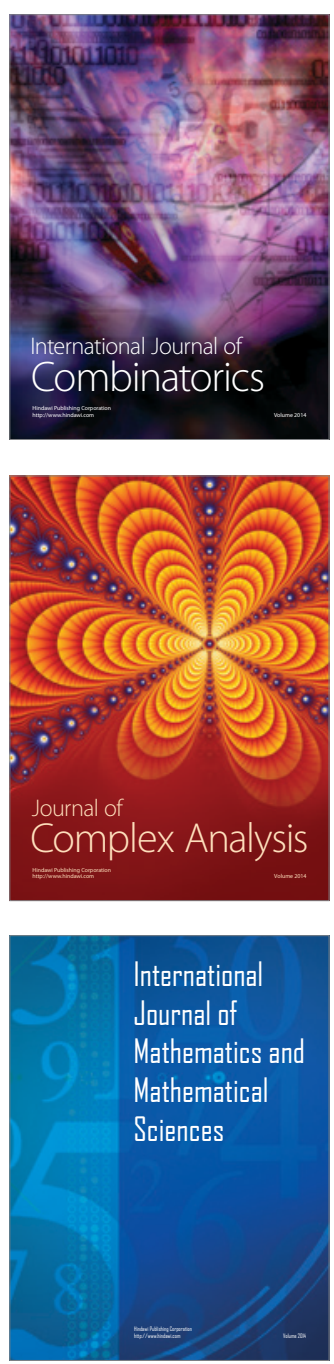
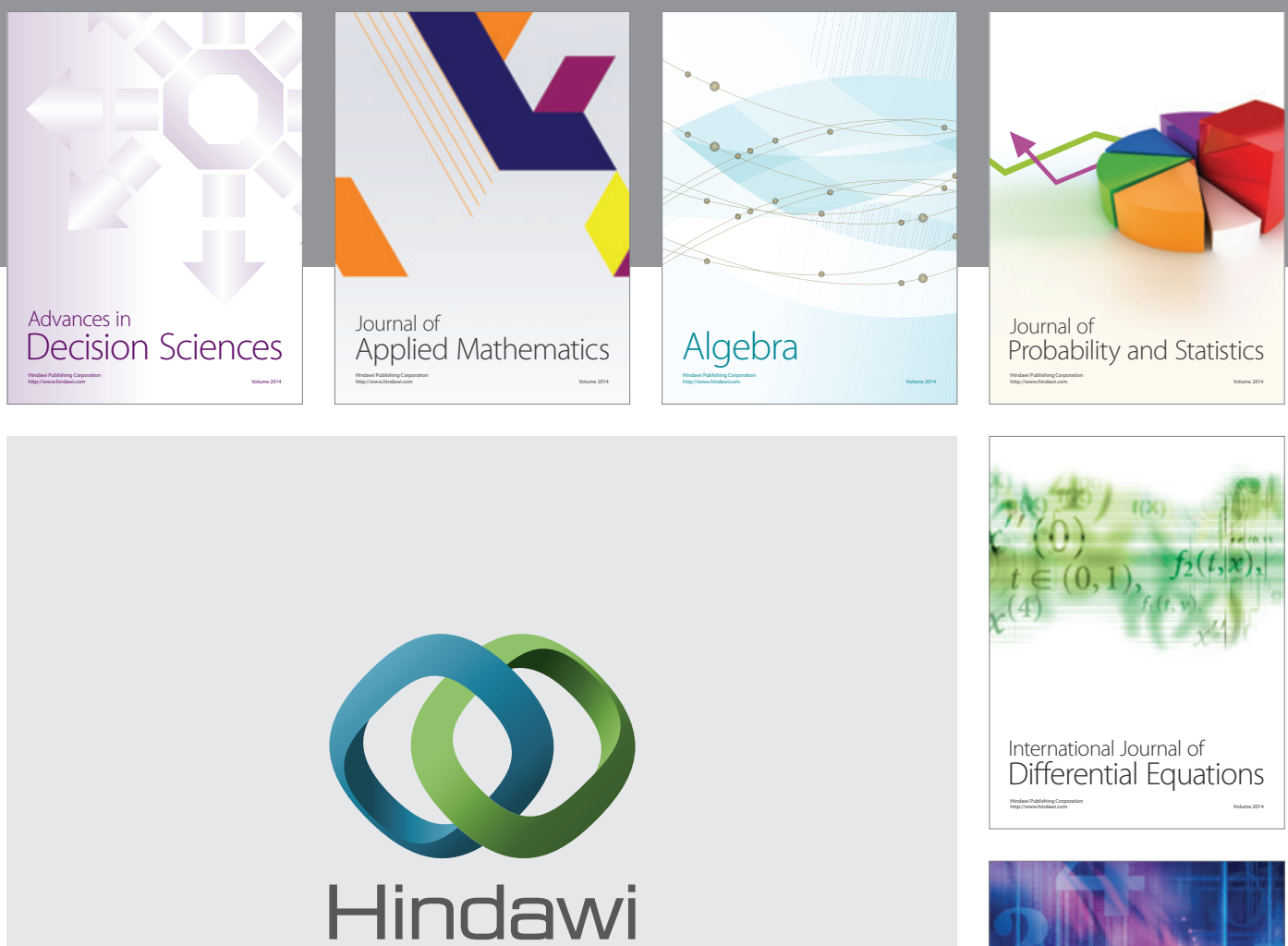

Submit your manuscripts at http://www.hindawi.com
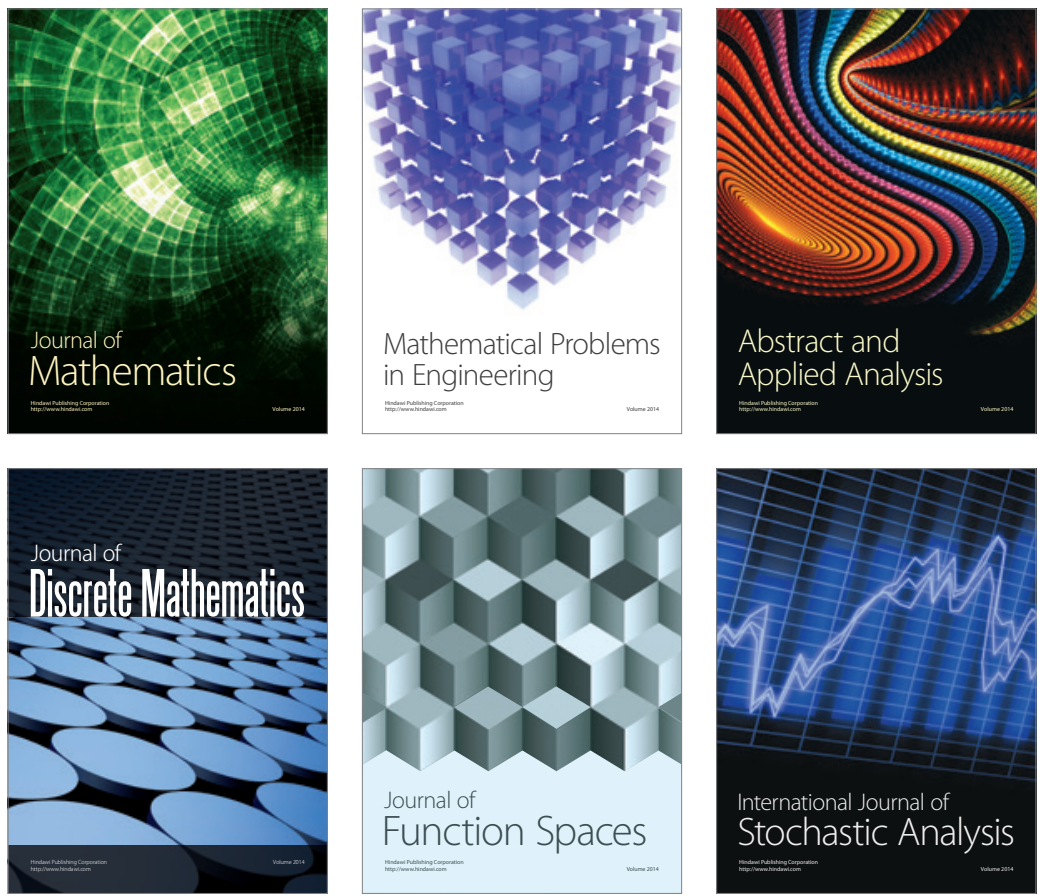

Journal of

Function Spaces

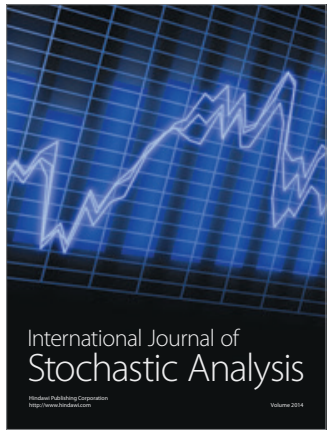

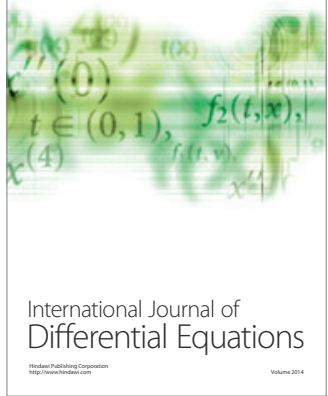
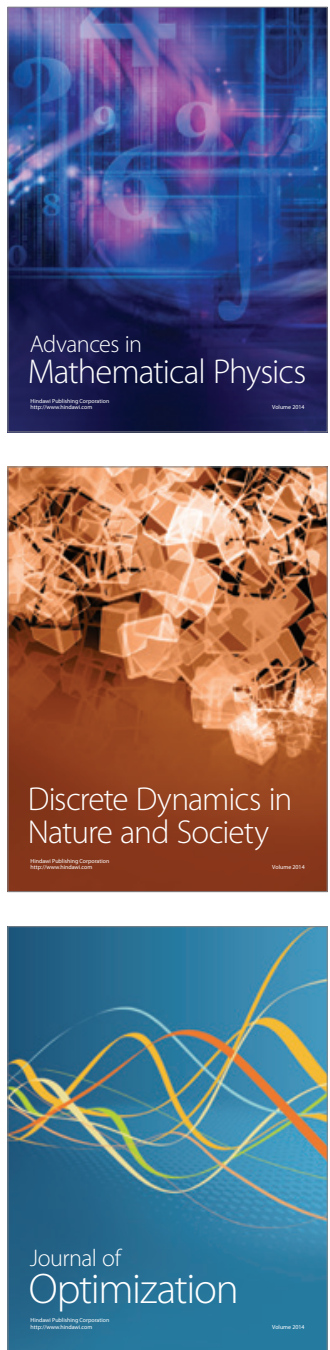